\title{
A rheological network model for the continuum anisotropic and viscoelastic behavior of soft tissue
}

Received: 2 December 2002/ Accepted: 2 May 2004/Published online: 23 July 2004

(C) Springer-Verlag 2004

\begin{abstract}
The mechanical behavior of soft tissue demonstrates a number of complex features including nonlinearity, anisotropy, viscoelasticity, and growth. Characteristic features of the time-dependent and anisotropic behavior are related to the properties of various components of the tissue such as fibrous collagen and elastin networks, large proteins and sugars attached to these networks, and interstitial fluid. Attempts to model the elastic behavior of these tissues based on assumptions about the behavior of the underlying constituents have been reasonably successful, but the essential addition of viscoelasticity to these models has been met with varying success. Here, a new rheological network model is proposed using, as its basis, an orthotropic hyperelastic constitutive model for fibrous tissue and a viscoelastic reptation model for soft materials. The resulting model has been incorporated into numerical and computational models, and is shown to capture the mechanical behavior of soft tissue in various modes of deformation including uniaxial and biaxial tension and simple shear.
\end{abstract}

\section{Introduction}

Biological soft tissues such as skin, tendon, and myocardium are known to exhibit very complex

\author{
J. E. Bischoff $(\square)$ \\ Department of Mechanical Engineering, \\ University of South Carolina, 300 South Main, \\ Columbia, SC 29208, USA \\ E-mail: bischoff@engr.sc.edu \\ Tel.: + 1-803-7770084 \\ Fax: $+1-803-7770106$ \\ E. M. Arruda $(\varangle) \cdot K$. Grosh $(\varangle)$ \\ Department of Mechanical Engineering, \\ University of Michigan, Ann Arbor, \\ MI 48105, USA \\ E-mail: arruda@umich.edu \\ E-mail: grosh@umich.edu
}

constitutive behaviors. Data from a variety of mechanical tests indicate the materials to be nonlinear, anisotropic, viscoelastic, and inhomogeneous, as well as susceptible to irreversible damage during the initial cycles of loading (termed "preconditioning" or "strain softening" in the biomechanics literature). Each of these constitutive features is closely linked with one or more aspects of the underlying microstructure; additionally, each microstructural feature (for example, the preferred alignment of collagen fibers in tendons) is the result of a continuous process of growth and adaptation of the tissue in response to external loads through cellular transduction. As such, a sound microstructural model for a tissue that successfully captures its salient constitutive characteristics can shed valuable insight into the underlying microstructure of the tissue, in both health and disease. With allowances for evolution of the material parameters in response to external loads and other stimuli, these models can be used to predict the response of the tissue to clinical interventions.

Attempts to model the quasi-static anisotropic nonlinear behavior of soft tissue have been reasonably successful, using a variety of phenomenological or microstructural approaches (Bischoff et al. 2002a; Billiar and Sacks 2000; Holzapfel et al. 2000; Humphrey et al. 1990; Horowitz et al. 1988; Demiray 1972; Veronda and Westmann 1970). However, modeling the time-dependent behavior of soft tissue adds an additional level of analytical and computational complexity, as the incorporation of viscoelasticity necessarily introduces additional material parameters. Also, because of the necessity for tracking the history of deformation in order to ensure equilibrium at the moment of interest, computational implementation of viscoelastic constitutive models can be demanding.

The underlying mechanisms for viscoelasticity in soft tissue depend on the tissue of interest, but can generally be categorized into three areas. First, as tissue is composed largely of fluid, there is substantial fluid flow through the fibrous network during deformation; this is the primary motivation for the development and use of 
multiphasic/poroelastic models (Mow et al. 1980; Simon et al. 1998). Additionally, there are many transient network connections in soft tissue (attachments between protein and sugar molecules and the collagen network, for example) that can be disrupted during deformation, but might reform upon removal of the load. Finally, individual components in the extracellular matrix (such as collagen and elastin fibers) are known to exhibit timedependent behavior (Fung 1981), thus contributing to the viscoelasticity of the tissue as a whole.

Several general techniques have been proposed for modeling the viscoelasticity of soft tissue including multiphasic models, hereditary integral formulations (quasilinear viscoelasticity), and state variable formulations. In addition to these approaches, rheological network models have been used extensively to model viscoelasticity in non-biological soft materials such as elastomers. In many such models, however, the inelastic strains are assigned independent evolution equations, and thus these models can be classified as state-variable models.

The multi phasic approach was initially developed for soft tissue by Mow et al. (1980) for modeling the timedependent behavior of cartilage; this approach has been widely used for the response of cartilage, employing various assumptions concerning the constituent solid and fluid phases (Soulhat et al. 1999). In particular, they proposed a biphasic model whereby the traditional governing equations of mechanics are expanded to allow for two independent phases (a solid matrix and interstitial fluid) which occupy and interact in the same continuum. Such a formulation is clearly applicable for modeling fluid flow in the tissue, but because of the additional physics, the governing field equations and constitutive models can become quite complex.

The hereditary integral formulation for modeling viscoelasticity in nonlinear materials was initially developed by Coleman and Noll (1961). It appears that this formulation was first utilized for modeling the timedependent behavior in soft tissue by Fung (1981) who coined the phrase "quasi-linear viscoelasticity" (QLV). The theory of QLV has since been adapted by numerous researchers to capture the response of a variety of soft tissues such as ligament (Provenzano et al. 2002) and tendon (Sverdlik and Lanir 2002; Pioletti and Rakotomanana 2000; Johnson et al. 1994; Woo et al. 1993). These models are characterized by an instantaneous elastic response (generally taken from a hyperelastic strain energy function proposed for the quasi-static behavior of the tissue) and a relaxation function that governs the "fading memory" of the current constitutive state on the deformation history. The models can take the form

$$
\boldsymbol{T}(t)=\hat{\boldsymbol{T}}^{(e)}(\mathbf{E}(t))+\int_{0}^{t} \hat{\boldsymbol{T}}^{(e)}(\boldsymbol{E}(t-\tau)) \frac{\partial G(\tau)}{\partial \tau} \mathrm{d} \tau
$$

where $\mathbf{T}$ and $\mathbf{E}$ are appropriate finite elasticity stress and strain measures, respectively, $\hat{\boldsymbol{T}}^{(e)}(\boldsymbol{E})$ is the instanta- neous (elastic) stress response of the material, and $G(\tau)$ is the relaxation function. Variations of the hereditary integral formulation arise from different forms of the instantaneous stress response, $\hat{\boldsymbol{T}}^{(e)}(\boldsymbol{E})$, or of the relaxation function, $G(\tau)$. Note that when $\mathbf{T}$ is taken to be the second Piola-Kirchhoff stress tensor, the elastic response, $\mathbf{T}^{(e)}$, is obtained from an appropriate anisotropic strain energy density function.

In the state variable approach for modeling nonlinear inelastic processes, a set of (state) variables is introduced to the problem. At each loading step, the values of these variables, as well as the appropriate measure of the mechanical deformation (e.g., the deformation gradient), must be determined. The governing equations of finite elasticity are augmented by evolution equations for the state variables, whereby the time derivatives of these variables are, in general, posited as functions of the local current strain or stress in the material, as well as of the current values of the state variables themselves (Holzapfel et al. 2002; Rubin and Bodner 2002).

The rheological network formulation of viscoelasticity, in which a discrete number of elastic and viscous elements are arranged in parallel and/or in series, is a subclass of the state variable formulation ${ }^{1}$. Though not often explicitly used to model the inelastic properties of tissue, the rheological network formulation has successfully been used to model the viscoelastic behavior of elastomers (Bergström and Boyce 1998). Such models can also be expanded to include other inelastic effects such as elastoplasticity (Miehe and Keck 2000). Viscoelastic models for soft tissue that employ a state-variable formulation (Holzapfel et al. 2002) or models of tissue growth that incorporate a decomposition of the deformation gradient (Taber and Humphrey 2001; Rodriguez et al. 1994) can be recast as rheological network models with appropriate constitutive equations for the elastic and inelastic components. In these models, the components of the deformation gradient in the inelastic (plastic, viscous, growth) network elements can be viewed as state variables.

Here, we propose a three-element (rheological) network constitutive model for capturing the nonlinear, viscoelastic, and orthotropic material behavior of biological soft tissue. The elastic elements are modeled using a nonlinear orthotropic constitutive law developed to simulate the orthotropic quasi-static nonlinear behavior of soft tissue. The viscous response is modeled using a reptation-based dashpot developed for application to the time-dependent behavior of elastomers. Using numerical and finite element simulations, the resulting model is shown to qualitatively capture the anisotropic, viscoelastic behavior that is characteristic of many types of soft tissue.

\footnotetext{
${ }^{1}$ Note that the use of "network" in this context refers to a combination of springs and dashpots, and not to an interconnected matrix of macromolecules or fibers that forms the basis for several microstructural models for elastomers and soft tissue (Bischoff et al. 2002b; Arruda and Boyce 1993).
} 


\section{Constitutive formulation}

Rheological network constitutive models in which parallel branches are included to model viscoelasticity, plasticity, damage, and other phenomena are frequently used to model elastomers (Bergström and Boyce 1998; Miehe and Keck 2000). In such formulations, the models for particular elastic and inelastic components are motivated by assumptions concerning the microstructural basis of the mechanical behavior, such as the entropics of long chain molecules to capture nonlinear elasticity (Treloar 1975). Time-dependent effects in monophasic tissue models are generally modeled using the theory of QLV (Fung 1981; Sverdlik and Lanir 2002; Puso and Weiss 1998), in which a hereditary integral is used to account for the time-dependent behavior. Here, adopting ideas from rubber elasticity, a rheological network formulation is proposed to capture the timedependent mechanical behavior of anisotropic viscoelastic soft tissue.

\subsection{Rheological representation}

The three-element rheological formulation used here is given schematically in Fig. 1, in which an elastic element is in parallel with a Maxwell fluid element. The forms of the constitutive relations for each particular element will be discussed subsequently; for now, they are given in general terms. The Cauchy stress tensor in each elastic element $\left(\mathbf{T}_{A}\right.$ and $\left.\mathbf{T}_{B}\right)$ is a function of the deformation gradient seen by that element ( $\mathbf{F}$ and $\mathbf{F}^{e}$, respectively). Additionally, the time derivative, $\dot{\boldsymbol{F}}^{v}$, of the deformation gradient in the viscous element is a constitutive function of the deformation gradient, $\mathbf{F}^{v}$, and the Cauchy stress tensor, $\mathbf{T}_{B}$, in that element. The total stress of the network is given by $\mathbf{T}=\mathbf{T}_{A}+\mathbf{T}_{B}$, and the deformation gradient is decomposed as $\mathbf{F}=\mathbf{F}^{e} \mathbf{F}^{v}$.

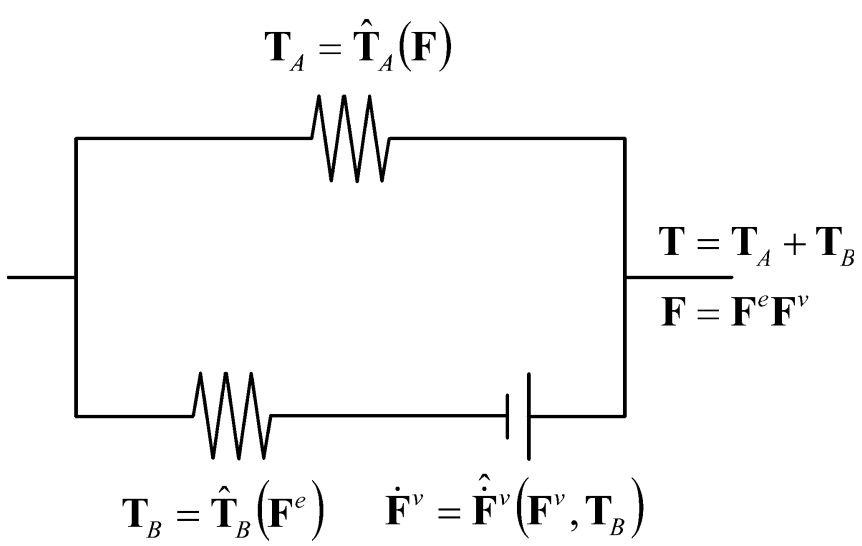

Fig. 1 Rheological network formulation of viscoelasticity that presupposes the material to respond elastically to finite deformation applied at infinitesimal strain rates

\subsection{Orthotropic eight-chain model}

The orthotropic eight-chain model has been developed to capture nonlinear elastic orthotropic constitutive properties of soft tissue such as skin and myocardium (Bischoff et al. 2002b). The model is derived from the response of a unit cell oriented in space according to the mutually orthogonal material directions $\mathbf{a}, \mathbf{b}$, and $\mathbf{c}$, with corresponding cell dimensions $a, b$, and $c$. The model is presented here using the deviatoric components of the deformation gradient, $\tilde{\boldsymbol{F}}=J^{-1 / 3} \boldsymbol{F}$ (where $\left.J=\operatorname{det} \mathbf{F}\right)$, and the right Cauchy-Green tensor, $\tilde{\boldsymbol{C}}=\tilde{\boldsymbol{F}}^{\mathrm{T}} \tilde{\boldsymbol{F}}$. The strain energy density function for the deviatoric model is given by

$$
\begin{aligned}
W= & W_{0}+\frac{n}{4} \sum_{i=1}^{4} w\left(\tilde{\rho}^{(i)}\right)-\frac{n}{4 P}\left(\frac{\mathrm{d} w(\tilde{\rho})}{\mathrm{d} \tilde{\rho}}\right)_{\tilde{\rho}=P} \ln \tilde{\lambda}_{a}^{a^{2}} \hat{\lambda}_{b}^{b^{2}} \tilde{\lambda}_{c}^{c^{2}} \\
& +\kappa[\cosh (J-1)-1]
\end{aligned}
$$

where $W_{0}$ is the strain energy density of the undeformed continuum; $n$ is a material parameter related to the fiber density; $\tilde{\rho}^{(i)}=\sqrt{\boldsymbol{P}^{(i) \mathrm{T}} \cdot \tilde{\boldsymbol{C}} \cdot \boldsymbol{P}^{(i)}}$ is the deformed length of a constituent fiber in the unit cell where $\mathbf{P}^{(i)}$ is the vector description of that fiber; $P=\sqrt{a^{2}+b^{2}+c^{2}} / 2$ is the undeformed length of a constituent fiber; $w(\tilde{\rho})$ is the strain energy density function of a single fiber; $\tilde{\lambda}_{a}, \tilde{\lambda}_{b}, \tilde{\lambda}_{c}$ are the deviatoric stretches along the principal material directions (given by, for example, $\tilde{\lambda}_{a}^{2}=\boldsymbol{a}^{\mathrm{T}} \cdot \tilde{\boldsymbol{C}} \cdot \boldsymbol{a}$ ); and $\kappa$ is a material parameter related to the bulk modulus of the continuum. This strain energy function is a function of a reduced set, $\left(\lambda_{a}, \lambda_{b}, \lambda_{c}, J\right)$, of the 15 strain invariants on which an orthotropic strain energy function can depend.

In this study, the freely jointed chain model will be used to characterize a single constituent fiber (Bischoff et al. 2002a). The fiber strain energy density function is defined as

$w(\rho)=w_{0}+k \Theta N\left(\frac{\rho}{N} \beta+\ln \frac{\beta}{\sinh \beta}\right)$

where $w_{0}$ is the strain energy density of an undeformed fiber; $k$ is Boltzmann's constant; $\Theta$ is absolute temperature; $N$ is the locking stretch of the chain and is related to the reference chain length according to the relation $N=P^{2} ;$ and $\beta=\mathcal{L}(\rho / N)$ where $\mathcal{L}(x)=\operatorname{coth}(x)-1 / x$ is the Langevin function. Note that this relation is of the form $w=\hat{w}(\rho)$, but it can be rewritten as a function of the deviatoric fiber length through the relation $\tilde{\rho}=J^{-1 / 3} \rho$.

Both elastic elements in the rheological model in Fig. 1 are modeled here using the deviatoric orthotropic eight-chain model. This assumption introduces ten independent material parameters (five parameters $n_{A}$, $a_{A}, b_{A}, c_{A}$, and $\kappa_{A}$ to characterize element $\mathrm{A}$, and five parameters $n_{B}, a_{B}, b_{B}, c_{B}$, and $\kappa_{B}$ to characterize element B). For certain connective tissues like skin, the parameters of element A can be interpreted in terms of a stable 
(collagenous) network in the tissue, and thus the five model parameters reflect the fiber density, orientation, and compressibility of the fibrous system. Similarly, the parameters of the reptating network (element B) can be understood in terms of a secondary network composed of other fibrous materials (elastin) and interstitial molecules. For both of these networks, it is assumed here that the material axes are aligned, i.e., $\mathbf{a}_{A}=\mathbf{a}_{B}$, thus ensuring orthotropic behavior of the continuum. By relaxing this constraint, a fully anisotropic continuum model can result.

\subsection{Bergström-Boyce model}

Bergström and Boyce developed a constitutive model for the viscoelastic properties of elastomers such as chloroprene and nitrile rubber (Bergström and Boyce 1998, 2001) based on the theory of reptation to account for the time-dependent slipping of constituent chains. Within the framework shown in Fig. 1, they prescribed flow in the viscous component according to the relation

$\widehat{D}^{v}=\dot{\gamma}_{B} N_{B}$

where $\widehat{D}^{v}=\boldsymbol{F}^{e} \dot{\boldsymbol{F}}^{v}\left(\boldsymbol{F}^{v}\right)^{-1}\left(\boldsymbol{F}^{e}\right)^{-1}$ is related to the rate of change of the viscous deformation gradient; $\dot{\gamma}_{B}$ is the reptation coefficient; and $\mathbf{N}_{B}$ is the direction of the stress that drives the viscous deformation and is given by $\boldsymbol{N}_{B}=$ $\boldsymbol{T}_{B} / \tau$ where $\boldsymbol{T}_{B}^{\prime}$ is the deviatoric stress on the viscous element and $\tau=\sqrt{\operatorname{tr}\left(\boldsymbol{T}_{B}^{\prime} \boldsymbol{T}_{B}^{\prime}\right)}$. Furthermore, considering the mechanics of the reptation coefficient, $\dot{\gamma}_{B}$, in Eq. 4, this coefficient is constitutively given as

$\dot{\gamma}_{B}=\dot{\gamma}_{0}\left(\bar{\lambda}^{v}-1+\varepsilon\right)^{C}\left(\frac{\tau}{\tau_{\text {base }}}\right)^{m}$

where $\bar{\lambda}^{v}=\sqrt{\operatorname{tr}\left(\boldsymbol{B}^{v}\right) / 3}$ and $\mathbf{B}^{v}=\mathbf{F}^{v}\left(\mathbf{F}^{v}\right)^{\mathrm{T}}$. According to Eq. 5, the constant material parameters in this model are $\dot{\gamma}_{0}, \varepsilon, C, \tau_{\text {base }}$, and $m$, though $\varepsilon$ was introduced solely for numerical difficulties that occur around $\bar{\lambda}^{v}=1$ (Bergström and Boyce 2001).

The Bergström-Boyce model is used to model the viscous element shown in Fig. 1. As such, five additional material parameters are introduced, and the complete orthotropic viscoelastic model is characterized by fifteen parameters.

\section{Results}

Five sets of results are presented here to demonstrate the features and capabilities of this model. First, the response of the model to uniaxial deformation along each of the three material axes is presented to illustrate the anisotropy of the model and the effects of preconditioning. Secondly, a parametric study of the model to uniaxial deformation along material axis a is shown, focusing on the effects of a few key parameters and of the strain rate. Thirdly, the response of the model to an instantaneously applied state of planar biaxial stretch is simulated, showing the stress relaxation features of the model. Fourth, the model is subjected to planar biaxial tension. These latter two sets of simulations are performed with an intent to simulate data in the literature obtained from tests on rabbit skin (Lanir and Fung 1974). Finally, finite element simulations of simple shear are presented and compared qualitatively with results from tests on rat septal myocardium (Dokos et al. 2000).

\subsection{Preconditioning}

Figure 2 demonstrates the response of the constitutive model to multiple cycles of uniaxial deformation along each of the three material axes $\mathbf{a}, \mathbf{b}$, and $\mathbf{c}$ (assumed to be aligned with the reference axes $\boldsymbol{x}_{1}, \boldsymbol{x}_{2}$, and $\boldsymbol{x}_{3}$, respectively). Each simulation was performed under constant strain rate control and a total of 20 cycles in each direction were simulated; the first cycle is the upper loop for each set and subsequent cycles follow the trend of the arrows in the figure. The material parameters used in these simulations are as follows: for the elastic elements, $n_{A}=10^{22} / \mathrm{m}^{3}, n_{B}=10^{23} / \mathrm{m}^{3}, a_{A}=a_{B}=1.3, b_{A}=b_{B}=1.15$, $c_{A}=c_{B}=1$, and $\kappa_{A}=\kappa_{B}=100 \mathrm{MPa}$; and for the viscous element, $C=-1, m=2, \tau_{\text {base }}=1 \mathrm{MPa}, \varepsilon=0.001$, and $\dot{\gamma}_{0}=1 / \mathrm{s}$. Additionally, the period of each loading/ unloading cycle was $T=1 \mathrm{~s}$.

The orthotropy of the response is dictated by the three unit cell dimensions $a, b$, and $c$ for each of the two elastic elements; the larger the value of the dimension, the stiffer the response of the model to deformation in that direction. The viscoelasticity is readily apparent in the initial cycle in each direction as there is substantial hysteresis. The material response in subsequent cycles becomes increasingly more compliant and the amount of

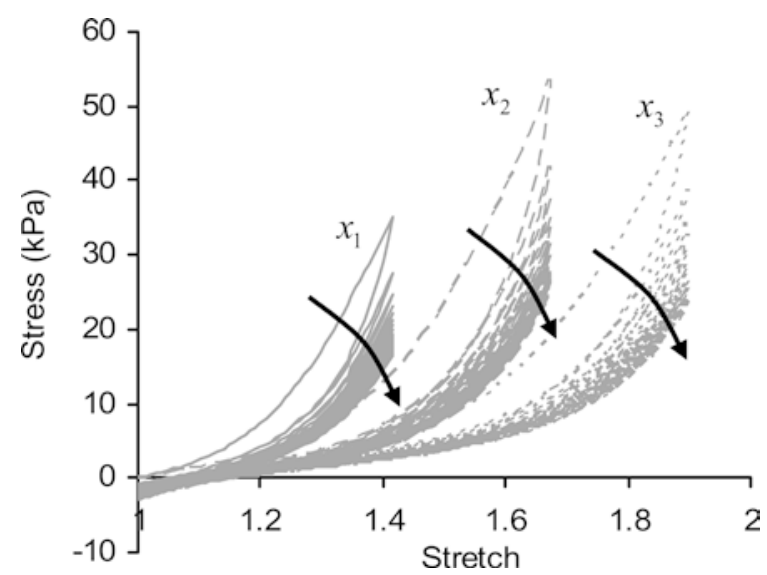

Fig. 2 Response of the constitutive model to uniaxial deformation along each of the three reference axes $\left(\boldsymbol{x}_{1}, \boldsymbol{x}_{2}\right.$, and $\left.\boldsymbol{x}_{3}\right)$. The material axes $\mathbf{a}, \mathbf{b}$, and $\mathbf{c}$ are aligned with the reference axes, and the simulations are performed under constant strain rate control. A total of 20 cycles for each test were simulated; the initial cycle is the upper loop in each set, and subsequent cycles follow the arrows in the figure. Material parameters are as indicated in the text, including $a_{A}=a_{B}=1.3, b_{A}=b_{B}=1.15$, and $c_{A}=c_{B}=1$ 
hysteresis significantly decreases; by the final cycle, there is little energy loss during loading and unloading. This feature has been previously observed in tests on skin, in which mechanical data demonstrate little hysteresis after many cycles of deformation (Brody et al. 1981).

\subsection{Uniaxial tension}

Figure 3 shows the effects of varying $n_{B}, \kappa_{B}$, and $T$ (the period of the deformation cycle) on the response of the model to uniaxial tension in the $\boldsymbol{x}_{1}$ direction. The tests are simulated under constant strain rate control and the test axis, $\boldsymbol{x}_{1}$, is assumed to be aligned with material axes $\mathbf{a}_{A}$ and $\mathbf{a}_{B}$. Unless explicitly stated in the figure, the parameters used to generate these results are the same as for Fig. 2, including $T=1 \mathrm{~s}$.

As shown in Fig. 3a, the stiffness of the model response increases as the stiffness of the series elastic element increases; similar results would be expected from varying the parallel elastic element stiffness. More significantly, as $n_{B}$ decreases (relative to $n_{A}$ ), the amount of viscous loss per cycle decreases because the reptating network freely distends in response to applied deformations. Note that the curves in Fig. 3a, corresponding to larger values of $n_{B}$, do not return to zero uniaxial stress immediately after the applied strain returns to zero; a negative (compressive) stress is required to return the material to zero strain. Over time, the magnitude of this compressive stress decreases and the initial undeformed (zero stress-zero strain) equilibrium state is again realized.

The response of the model stiffens significantly as $\kappa_{B}$ increases (Fig. 3b), indicating premature locking. For large values of the bulk modulus $\left(\kappa_{B}=10 \mathrm{MPa}\right.$ and above) the model response ceases to change as the incompressible limit has effectively been reached (note that the shear modulus for the series elastic element is approximately $400 \mathrm{~Pa}$ ).

Figure $3 \mathrm{c}$ shows the effects of varying the period of deformation, $T$, or, equivalently, the applied strain rate, $\dot{e}$, on the response of the model to uniaxial deformation. The longest period of deformation shown here ( $T=100 \mathrm{~s}$, corresponding to a strain rate of $\dot{e}=0.01 / \mathrm{s}$ ) does not approximate a quasi-static simulation as there is still noticeable hysteresis. Over the several decades of strain rates shown in Fig. 3c, the response of the model stiffens with increasing strain rate (decreasing $T$ ), indicating a definitive rate dependence. However, over these decades, the amount of hysteresis does not vary as much, particularly in the range $1 \mathrm{~s}<T<100 \mathrm{~s}$; such behavior is consistent with the known rate independence of hysteresis in soft tissue (Fung 1981).

\subsection{Stress relaxation}

Figure 4 shows the response of the model to biaxial stress relaxation validated against data from Lanir and Fung (1974). These data were obtained by subjecting a (a)

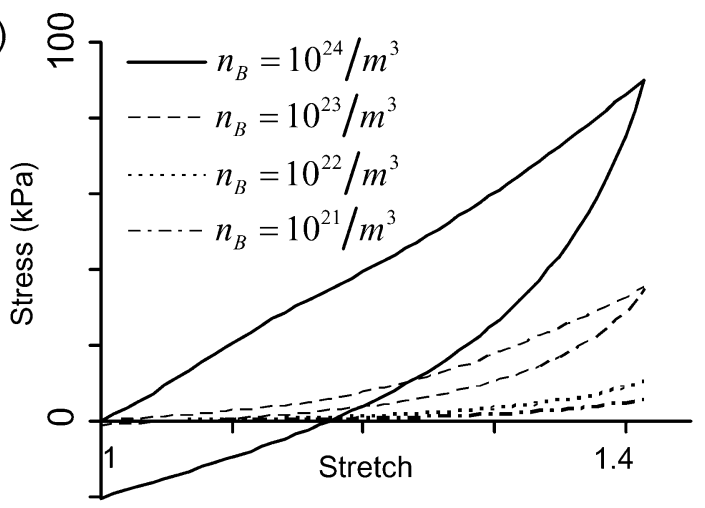

(b)

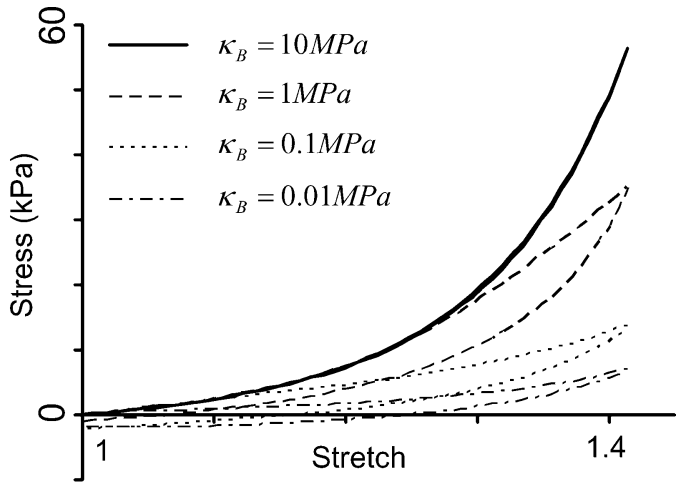

(c)

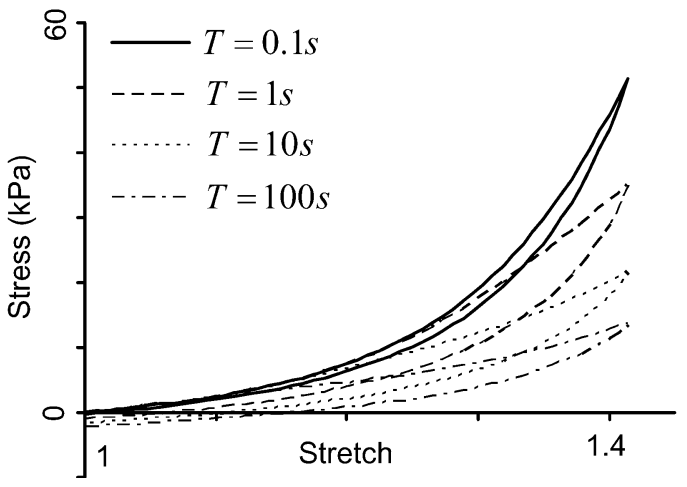

Fig. 3a-c Parametric study on the effects of various parameters on the response of the model to uniaxial deformation (constant strain rate for one loading/unloading cycle) along the $\boldsymbol{x}_{1}$ axis. a $n_{B}$, proportional to the initial stiffness of the series elastic element. b $\kappa_{B}$, the bulk modulus in the series elastic element. c $T$, the period of the deformation. Values of the remaining material parameters are as given in the text

square piece of rabbit skin from the belly of the animal to an instantaneous planar biaxial stretch $\left(\lambda_{1}=1.632\right.$, $\lambda_{2}=1$ ), which was subsequently maintained for an extended period of time. The reference axes, $\boldsymbol{x}_{1}$ and $\boldsymbol{x}_{2}$, were aligned with the length of the animal and the width of the animal, respectively, and thus it is reasonable to assume (as was done here) that these axes are also aligned with material directions a and $\mathbf{b}$. Selected data were digitized and reproduced and are shown here in Fig. 4 as symbols. Note that, although it is generally 


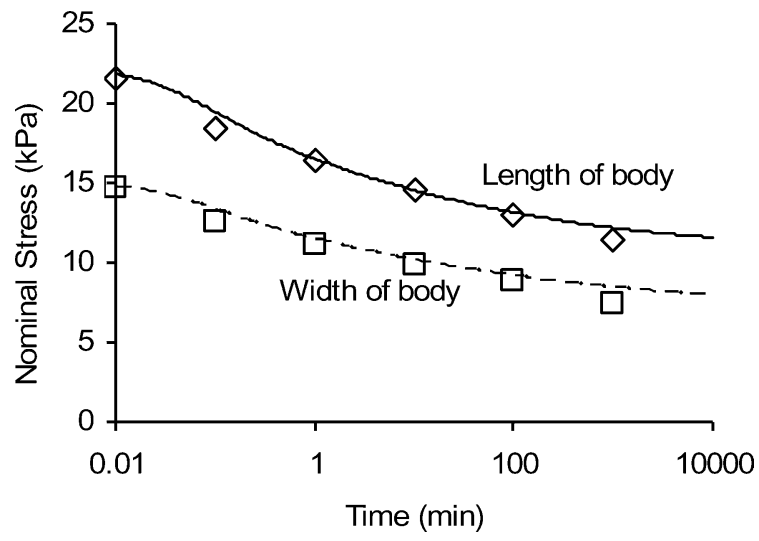

Fig. 4 Stress relaxation following a step biaxial stretch on rabbit skin. Data (shown using symbols) were obtained by loading a square piece of excised skin from the belly of a rabbit in a state of planar biaxial tension with $\lambda_{1}=1.632$ and $\lambda_{2}=1$ (Lanir and Fung 1974). For these tests, the $\boldsymbol{x}_{1}$ direction was oriented along the length of the animal and the $\boldsymbol{x}_{2}$ direction was oriented along the width of the animal. Simulation results are shown in the figure as lines, and were obtained using material parameters as given in the text

true that the anatomical axes are aligned with the planar material axes, there is significant variability of the degree of collagen alignment in these directions and hence a similar variability in the anisotropic stiffnesses (Lanir and Fung 1974).

The response of the model to this deformation was calculated by assuming an instantaneous increase in the biaxial stretch to its final value, such that the viscous element was initially rigid. Material parameters used in the simulation are as follows: for both of the elastic elements $n=10^{23} / \mathrm{m}^{3}, \quad a=1.4, \quad b=1.6, \quad c=1, \quad$ and $\kappa=100 \mathrm{MPa}$; and for the viscous element $C=-1.5$, $m=1.8, \tau_{\text {base }}=60 \mathrm{MPa}, \varepsilon=0.001$, and $\dot{\gamma}_{0}=1 / \mathrm{s}$. Simulation results are shown in the figure as lines and demonstrate good consistency with the experimental data.

\subsection{Biaxial tension}

Figure 5 shows data (dashed lines) obtained from biaxial tension tests on rabbit skin (Lanir and Fung 1974), along with simulation results (solid lines) using the constitutive model. The two sets of experimental data were obtained from distinct tests: to generate the data indicated as "along length of body," a constant stretch rate was applied along the body length while restricting any in-plane lateral contraction, and vice versa for the data indicated as "along width of body." These data demonstrate orthotropy in loading and unloading, significant and sharp strain hardening (locking) behavior, and large viscous losses immediately following the reversal of strain.

To simulate these experiments, the anatomical directions "length" and "width" were assumed to be aligned with reference axes $\boldsymbol{x}_{1}$ and $\boldsymbol{x}_{2}$ and material axes $\mathbf{a}$ and $\mathbf{b}$, respectively. An applied stretch rate of

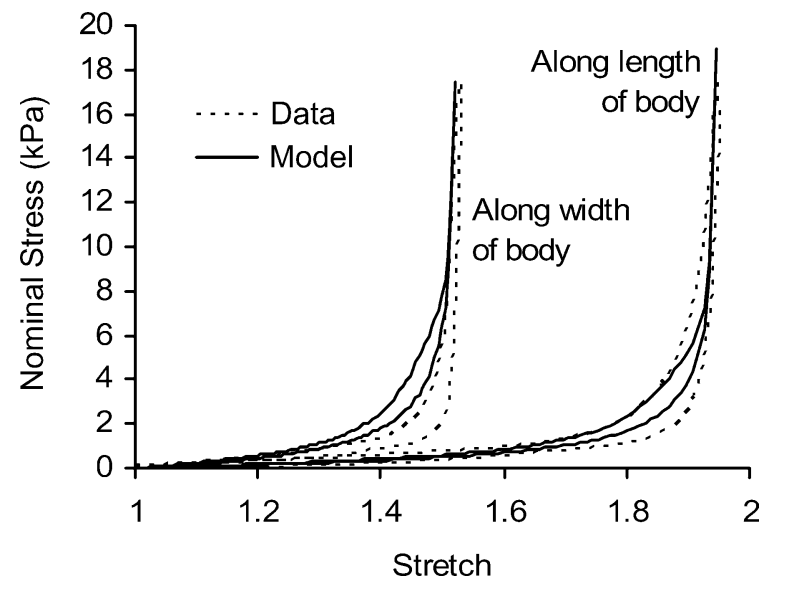

Fig. 5 Experimental data from planar biaxial tension tests on excised rabbit skin (digitized and reproduced from Lanir and Fung 1974) and model predictions. In each test, a stretch was applied to one of the in-plane material directions while the transverse in-plane dimension was held fixed. The anatomical directions "Along length of body" and "Along width of body" were assumed to be aligned with the $\boldsymbol{x}_{1}$ and $\boldsymbol{x}_{2}$ axes, respectively, and indicate the direction along which the positive strain was applied. Data are indicated using dashed lines, simulation results are drawn using solid lines. Material parameters are as given in the text

$\dot{\lambda}=0.00571 / \mathrm{s}$ was used, corresponding to a displacement rate of $\dot{d}=2 \mathrm{~mm} / \mathrm{s}$, based on sample dimensions reported in the literature. Material parameters that were varied in order to generate these fits are as follows: for the parallel elastic element, $n_{A}=10^{21} / \mathrm{m}^{3}, a_{A}=1.52$, $b_{A}=2.2, \quad c_{A}=0.5 ;$ for the series elastic element, $n_{B}=4 \times 10^{21} / \mathrm{m}^{3}$; and for the viscous element, $C=-1.8$, $m=5$, and $\tau_{\text {base }}=120 \mathrm{kPa}$. The unit cell dimensions of the series elastic element were set to be equal to those of the parallel elastic element; the bulk moduli of both elastic elements were set to be greater than the corresponding shear moduli by a factor of six (thus, ensuring near incompressibility); and the remaining viscous parameters were held fixed as $\varepsilon=0.001$ and $\dot{\gamma}_{0}=1 / \mathrm{s}$.

A similar degree of anisotropy between simulation results and experimental data is seen in Fig. 5, though the data demonstrate more severe locking behavior than can be captured by the orthotropic elements in the model. The nature of the hysteresis for each of the two data sets is captured by the model in that most of the viscous losses occur around the locking elbow. However, the model underestimates the total amount of hysteresis, which could be due to constitutive model limitations or it could be a consequence of inaccurate simulation assumptions (such as the applied stretch rate).

It is worth noting that the data in Figs. 4 and 5 were obtained from distinctly different animals. This may be seen by comparing the stress levels in the two data sets at equivalent strains. In Fig. 4, at a stretch state of $\lambda_{1}=1.632, \lambda_{2}=1$ (maintained throughout the test) the length-wise stress relaxes from $22 \mathrm{kPa}$ to $13 \mathrm{kPa}$. In Fig. 5, for the same stretch state, the stress level is only about $0.7 \mathrm{kPa}$. In modeling these two types of tests therefore, it is not surprising that the parameter values 
are markedly different; in particular, values of the shear moduli, bulk moduli, and $\tau_{\text {base }}$ used in Fig. 5 are roughly two orders of magnitude smaller than those used in Fig. 4. This variability is not a shortcoming of the model; the differences in the data are accurately reflected in the model parameters and underline the recognized need to acquire a complete set of data from a single tissue specimen when validating complex material models.

\subsection{Simple shear}

Data from simple shear tests that demonstrate material anisotropy and viscoelasticity have recently been presented in the literature (Dokos et al. 2000) in which a rectangular piece of rat septal myocardium was fixed between two plates and sheared in two mutually orthogonal directions. Representative data from these experiments were digitized and are reproduced in Fig. 6, along with a schematic of the test with sample dimensions and test axis orientation. "Posterior-anterior" (PA) and "apex-base" (AB) denote two mutually orthogonal anatomical directions and are used in the figure to represent the orientation of the applied shear as follows. Assuming that the PA and $\mathrm{AB}$ directions correspond with the $\boldsymbol{x}_{1}$ and $\boldsymbol{x}_{3}$ test axes, respectively, then, in the PA test, the upper surface in Fig. 6 is displaced along the $\boldsymbol{x}_{1}$ (PA) direction; in the $\mathrm{AB}$ test, the upper surface is displaced along the $\boldsymbol{x}_{3}(\mathrm{AB})$ direction. Note that the anatomically-based orientations "posterioranterior" and "apex-base" do not necessarily correspond with principal material directions as the fiber orientation in the septum is highly irregular and inhomogeneous.

The experimental protocol was simulated using ABAQUS, a commercially available general purpose

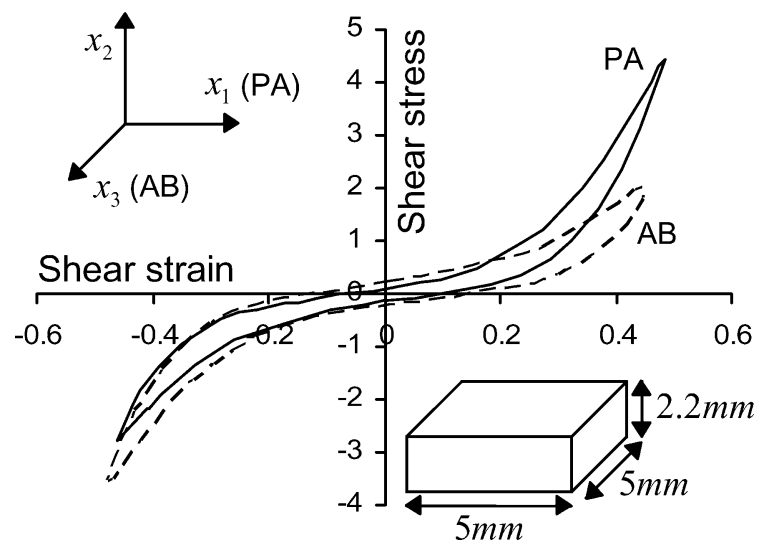

Fig. 6 Constitutive data obtained from simple shear tests on rat septal myocardium, digitized from Dokos et al. (2000) and reproduced here. The test axis $\boldsymbol{x}_{1}$ corresponds to the anatomical posterior-anterior (PA) direction; the test axis $\boldsymbol{x}_{3}$ corresponds to the anatomical apex-base (AP) direction. The PA simple shear state occurs when the upper surface is displaced in the $\boldsymbol{x}_{1}$ direction; the $\mathrm{AB}$ simple shear state occurs when the upper surface is displaced in the $\boldsymbol{x}_{3}$ direction. Specimen dimensions are as indicated in the figure finite element software package that allows for incorporation of novel constitutive models (Hibbitt, Karlsson \& Sorensen, Inc. 2001). The computational model (size $5 \times 2.2 \times 5 \mathrm{~mm}$ ) was the same as in the experiments. The upper surface of the tissue was displaced (sinusoidal signal, amplitude $1.1 \mathrm{~mm}$, period $30 \mathrm{~s}$ ) in two orthogonal directions $\left(x_{1}\right.$ and $\left.x_{3}\right)$ for a fixed material orientation. The material axes are not assumed to be aligned with the test axes because the data in Fig. 6 demonstrate a directional dependence of the stresses; that is, for a given orientation (PA or $\mathrm{AB}$ ), the magnitudes of the stresses obtained for a given shear displacement in the positive and negative directions are not equivalent. Accordingly, the material axes are defined by $\mathbf{a}=(0.9924, \quad-0.1228, \quad 0.0076), \quad \mathbf{b}=(0.1228, \quad 0.9848$, $-0.1228)$, and $\mathbf{c}=(0.0076,0.1228,0.9924)$, corresponding to a $10^{\circ}$ rotation of the material axes about the line $x=z, y=0$. Note that this is a significant assumption about the structure of the tissue; given the size of the tissue and the fact that it was excised from the septal wall, it is likely that the material structure is heterogeneous, both in terms of material axes and parameters. Such structural data is essential to precise fitting of the data, and, given the lack of this data, only a qualititative fit is sought here.

A 125 -element $(5 \times 5 \times 5)$ model was created to simulate these tests using twenty-noded quadratic hybrid brick elements with reduced integration. Nonlinear deformations and rotations were permitted. The lack of alignment between the material axes and the reference (test) axes prevented symmetry arguments from being used to reduce the model size. Material parameters used in these simulations are as follows: for the elastic elements $n_{A}=2 \times 10^{15} / \mathrm{mm}^{3}, \quad n_{B}=1 \times 10^{15} / \mathrm{mm}^{3}$, $a_{A}=a_{B}=2.5, \quad b_{A}=b_{B}=1.75, \quad c_{A}=c_{B}=2, \quad$ and $\kappa_{A}=\kappa_{B}=100 \mathrm{MPa}$; and for the viscous element, $C=-1$, $m=3, \tau_{\text {base }}=10 \mathrm{kPa}, \varepsilon=0.01$, and $\dot{\gamma}_{0}=1 / s$. Stress versus strain results extracted from the simulation are shown in Fig. 7. Two cycles of deformation were plotted

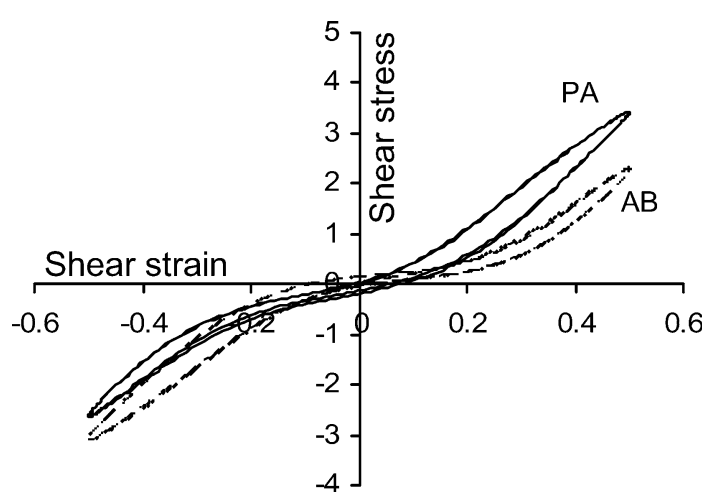

Fig. 7 Shear stress versus shear strain data extracted from finite element simulations of simple shear using the orthotropic viscoelastic model. Two cycles are simulated for each test (PA and AB) and both cycles are shown in the figure. Subsequent cycles are not noticeably different from the second cycle. Material parameters are as indicated in the text 
for each test, as can be seen by the slight amount of preconditioning (strain softening). Five cycles per test were simulated, but there was negligible difference between the second cycle and subsequent cycles.

Many of the same features of the constitutive data shown in Fig. 6 have been replicated in Fig. 7. The model response is clearly anisotropic as shear strains in the $\mathrm{PA}$ and $\mathrm{AB}$ directions do not produce the same stresses; this is a direct consequence of the orthotropic material parameters $(a \neq b \neq c)$. Additionally, the response of the model to positive shear in the PA direction is stiffer than the response of the model to positive shear in the $\mathrm{AB}$ direction; this result is opposite to what happens in negative shear, when shear in the $A B$ direction is seen to produce the larger (negative) stress. Both of these trends are consistent with the experimental data. The reason for this directional dependence in the computational model is the slightly skewed alignment of the material axes relative to the testing axes in the model formulation; the experimental result could be a reflection of this same homogeneous misalignment or to a spatially varying orientation of the material axes within the test sample.

\section{Discussion}

A new anisotropic viscoelastic constitutive model for soft tissue has been developed using as its basis (1) a five parameter orthotropic hyperelastic constitutive model developed for the quasi-static behavior of soft tissue, and (2) a viscous reptation-based model developed for application to soft (isotropic) materials, such as elastomers. The resulting constitutive model is orthotropic and viscoelastic and, as such, is applicable to tissues such as myocardium and skin, which demonstrate time-dependent effects and this class of material symmetry. The model is of the state-variable form whereby the nine components of the viscous deformation gradient are treated as independent state variables with appropriate evolution equations in terms of the current deformation and stresses in the material.

Several models for the nonlinear viscoelastic properties of soft tissue with varying levels of material symmetry have recently appeared. Sverdlik and Lanir (2002) introduced a thirteen-parameter QLV-based model to capture the one-dimensional time-dependent properties (including preconditioning) of tendons. Rubin and Bodner (2002) utilized a fourteen-parameter model to simulate the response of a soft tissue with a single class of fibers. Holzapfel and Gasser (2001) simulated the response of soft tissue with two classes of fibers using a state-variable formulation with at least twelve independent material parameters, depending on the number of relaxation processes required. Here, a 15-parameter model for the nonlinear time-dependent response of a fully orthotropic tissue with three independent fiber classes is proposed.
The majority of the 15 parameters describe the two elastic elements in the rheological model, each of which requires five parameters according to the orthotropic hyperelastic model previously developed (Bischoff et al. 2002a). However, not all 15 parameters are freely varied. Firstly, the two bulk moduli, $\kappa_{A}$ and $\kappa_{B}$, are, in general, set to be many orders of magnitude greater than the corresponding shear moduli to ensure near incompressibility. Secondly, the viscous parameter, $\varepsilon$, is an artificial parameter introduced to avoid numerical difficulties about zero strain (Bergström and Boyce 2001), and the viscous parameter, $\dot{\gamma}_{0}$, is a toggle switch that activates the time-dependency in the model. Additionally, it was not required to distinguish between the unit cell dimensions of the two elastic elements (i.e., it was sufficient to set $a_{A}=a_{B}, b_{A}=b_{B}$, and $c_{A}=c_{B}$ ) when fitting the model to experimental data. Thus, only eight independent parameters were varied to capture anisotropic and viscoelastic characteristics from a variety of tests on tissue, including uniaxial tension, planar biaxial tension, and simple shear.

The orthotropic elastic elements and the three-element rheological formulation have microstructural underpinnings that can, in theory, be used to relate parameters to structures of certain tissues such as skin and other connective tissue. In these cases, the parameters are not merely empirical constants but, rather, reflect the underlying architecture. In particular, for tissues like skin, the parameters in the parallel elastic element reflect properties of a stable collagen network, known to act as the mechanical backbone of many types of soft tissue; the fiber density, $n_{A}$, reflects the amount of collagen material present and the orthotropic aspect ratios, $a_{A}, b_{A}$, and $c_{A}$, represent the degree of anisotropy of the fibers. The series elastic element (with a subscript $B$ ) represents a secondary network; depending on the particular tissue type, this could be the network of elastin fibers, or could represent the transient connections between various protein and sugar molecules and the more permanent collagen network. These comments are not valid for myocardium, which has a very different structure; however, the model is still able to capture mechanical data (Fig. 7) using parameters that are, in this case, strictly empirical.

Regardless of any microstructural underpinnings to this new constitutive formulation, simulations of tissue deformation using this model indicate its ability to fit the mechanical response of soft tissue. The well known "rate-independent" response of soft tissue, whereby the hysteresis observed in the tissue response over several orders of magnitude of strain rate is relatively constant (Fung 1981), is successfully predicted, as shown in Fig. 3c. (Note that, in the general use of the term, tissue response is rate-dependent, as the constitutive response clearly stiffens with increased strain rate; classification of tissue as being rate-independent applies only when considering the amount of hysteresis during cyclic loading.) Representative data from the literature have been digitized and reproduced here (Figs. 4, 5, and 7). Results 
from numerical or computational simulations designed to closely match the experimental protocols that were used to obtain these data indicate a good agreement with the data. Better fits would likely be obtained with information about the material axes' orientations in the test specimens.

A significant drawback to this formulation, and indeed to any rheological (spring-dashpot) network formulation that endeavors to capture both anisotropic and viscoelastic features, is the large number of independent material parameters that must be specified. Whereas the orthotropic hyperelastic constitutive model which is used here to model the two elastic elements does a good job capturing anisotropic data from quasi-static tests of soft tissue using a small number of parameters (five), an additional ten parameters are used here to enable the model to capture the time-dependent effects. The effects of each of the parameters can likely be seen from an appropriate set of tests encompassing a variety of deformation modes and histories. For example, the five constants for the parallel elastic network could be extracted from a series of quasi-static uniaxial or biaxial tension tests. However, the model is overparameterized for most sets of constitutive data in the literature, as evidenced by the minimal influence of several parameters when simulating the tests in Figs. 4, 5, and 7; fits to these data did not require independent variation of the unit cell dimensions of the two elastic elements. Nevertheless, due to the success of the model in predicting reasonable behavior as discussed above, this model serves as a suitable starting point for expanding the orthotropic hyperelastic model into the viscoelastic domain.

It is important to acknowledge a number of issues associated with the implementation of this model into numerical or computational code. Firstly, the strainhardening (locking) behavior in the elastic elements is governed by the behavior of the inverse Langevin function, which is not defined for arguments greater than unity. Numerical problems can, thus, arise when a trial solution for the local deformation gradient results in a chain stretch greater than its maximally extended length. This problem can be avoided by using a series representation of the inverse Langevin function, which is continuously defined for all chain stretches; however, the sharpness of the locking behavior is compromised (results shown here were not generated using a series representation). Secondly, there is currently no established maximum allowable time step for this type of model that ensures convergence in an explicit formulation; time steps are, thus, assigned in a more or less random fashion and their suitability only determined well into the simulation when results diverge or remain well behaved. Thirdly, the ellipticity of the anisotropic elastic strain energy function is under investigation and has not yet been established, and, thus, the presence of potentially unstable deformation states that can confound numerical analysis remains unknown at this time.
In summary, a new orthotropic viscoelastic model has been formulated based on a three-element rheological network model with nonlinear elastic and viscous elements appropriate for large deformations. The resulting model can be cast in state-variable form, whereby evolution equations for nine state variables (representing the components of the viscous deformation gradient) are required in addition to the traditional equations of equilibrium. Using appropriate numerical and computational techniques, the model has been shown to successfully mimic the characteristic behavior of a variety of tissue types in several modes of deformation. Because of the elemental nature of the model, variations to accommodate materials of different symmetry classes (transverse isotropy as is appropriate for tendon and ligament, for example) can be easily incorporated, as well as altered viscous formulations.

\section{References}

Arruda EM, Boyce MC (1993) A three-dimensional constitutive model for the large stretch behavior of rubber elastic materials. J Mech Phys Solids 41:389-412

Bergström JS, Boyce MC (1998) Constitutive modeling of the large strain time-dependent behavior of elastomers. Mech Mate 32:627-644

Bergström JS, Boyce MC (2001) Constitutive modeling of the timedependent and cyclic loading of elastomers and application to soft biological tissues. Mech Mater 33:523-530

Billiar KL, Sacks MS (2000) Biaxial mechanical properties of the natural and glutaraldehyde treated aortic valve cusp. Part II: a structural constitutive model. J Biomech Eng 122:1-9

Bischoff JE, Arruda EM, Grosh K (2002a) A microstructurally based orthotropic hyperelastic constitutive law. J Appl Mech 69:570-579

Bischoff JE, Arruda EM, Grosh K (2002b) Orthotropic hyperelasticity in terms of an arbitrary molecular chain model. J Appl Mech 69 (4):198-201

Brody GS, Peng TJ, Landel RF (1981) The rheological properties of human skin and scar tissue. In: Marks R, Payne PA (eds) Bioengineering and the skin. MTP Press, Lancaster, Boston, pp 147-160

Coleman BD, Noll W (1961) Foundations of linear viscoelasticity. Rev Mod Phys 33(2):239-249

Demiray H (1972) A note on the elasticity of soft biological tissues. J Biomech 5:309-311

Dokos S, LeGrice IJ, Smaill BH, Kar J, Young AA (2000) A triaxial-measurement shear-test device for soft biological tissues. J Biomech Eng 122:471-478

Fung YC (1981) Biomechanics: mechanical properties of living tissue. Springer, Berlin Heidelberg, New York

Hibbitt, Karlsson \& Sorensen, Inc (2001) ABAQUS/Standard user's manual, version 6.2. Hibbitt, Karlsson \& Sorensen, Pawtucket, Rhode Island

Holzapfel GA, Gasser TC (2001) A viscoelastic model for fiberreinforced composites at finite strains: continuum basis, computational aspects and applications. Comput Meth Appl Mech Eng 190:4379-4403

Holzapfel GA, Gasser TC, Ogden RW (2000) A new constitutive framework for arterial wall mechanics and a comparative study of material models. J Elasticity 61(1):1-48

Holzapfel GA, Gasser TC, Stadler M (2002) A structural model for the viscoelastic behavior of arterial walls: continuum formulation and finite element analysis. Eur J Mech A-Solid $21: 441-463$ 
Horowitz A, Lanir Y, Yin FCP, Perl M, Sheinman I, Strumpf RK (1988) Structural three-dimensional constitutive law for the passive myocardium. J Biomech Eng 110:200-207

Humphrey JD, Strumpf RK, Yin FCP (1990) Determination of a constitutive relation for passive myocardium. Part I: a new functional form. J Biomech Eng 112:333-339

Johnson GA, Tramaglini DM, Levine RE, Ohno K, Choi NY, Woo, SLY (1994) Tensile and viscoelastic properties of human patellar tendon. J Orthop Res 12:796-803

Lanir Y, Fung YC (1974) Two-dimensional mechanical properties of rabbit skin. Part II: experimental results. J Biomech 7:171-182

Miehe C, Keck J (2000) Superimposed finite elastic-viscoelasticplastoelastic stress response with damage in filled rubbery polymers. Experiments, modelling and algorithmic implementation. J Mech Phys Solids 48:323-365

Mow VC, Kuei SC, Lai WM, Armstrong CG (1980) Biphasic creep and stress relaxation of articular cartilage in compression: theory and experiments. J Biomech Eng 102:73-84

Pioletti DP, Rakotomanana LR (2000) Non-linear viscoelastic laws for soft biological tissues. Eur J Mech A-Solid 19:749-759

Provenzano PP, Lakes RS, Corr DT, Vanderby R Jr (2002) Application of nonlinear viscoelastic models to describe ligament behavior. Biomech Model Mechanobiol 1:45-57

Puso MA, Weiss JA (1998) Finite element implementation of anisotropic quasi-linear viscoelasticity using a discrete spectrum approximation. J Biomech Eng 120:62-70
Rodriguez EK, Hoger A, McCulloch AD (1994) Stress-dependent finite growth in soft elastic tissues. J Biomech 27(4):455-467

Rubin MB, Bodner SR (2002) A three-dimensional nonlinear model for dissipative response of soft tissue. Int J Solids Struct 39:5081-5099

Simon BR, Kaufmann MV, McAfee MA, Baldwin AL, Wilson LM (1998) Identification and determination of material properties for porohyperelastic analysis of large arteries. J Biomech Eng 120:188-194

Soulhat J, Buschmann MD, Shirazi-Adl A (1999) A fibril-networkreinforced biphasic model of cartilage in unconfined compression. J Biomech Eng 121:340-347

Sverdlik A, Lanir Y (2002) Time-dependent mechanical behavior of sheep digital tendon, including the effects of preconditioning. J Biomech Eng 124:78-84

Taber LA, Humphrey JD (2001) Stress-modulated growth, residual stress, and vascular heterogeneity. J Biomech Eng 123:528-535

Treloar LRG (1975) The physics of rubber elasticity. Clarendon Press, Oxford

Veronda DR, Westmann RA (1970) Mechanical characterization of skin: finite deformations. J Biomech 3:111-124

Woo SLY, Johnson GA, Smith BA (1993) Mathematical modeling of ligaments and tendons. J Biomech Eng 115:468-473 